\title{
Gradual Efficiency Improvement through a Sequence of Targets
}

\author{
Akram Dehnokhalaji; Narges Soltani ${ }^{\dagger}$
}

January 2018

\begin{abstract}
The goal in efficiency analysis is not only to evaluate a Decision Making Unit (DMU) performance, but also to find an efficient target which provides information on inputs reduction and outputs increment values that are necessary to remove inefficiencies for each inefficient DMU. In Data Envelopment Analysis (DEA) the target unit is located on the efficient frontier and possibly far from the unit under assessment. Therefore, in practice performance improvement seems to be disappointing or even impossible to achieve in only one step for some inefficient DMUs. In this regard, finding intermediate targets is of great importance in benchmarking literature. In this paper we find a sequence of targets instead of a single target for each inefficient unit. In our method, the intermediate target at each step has three properties:(I) the intermediate targets and the unit under evaluation are all similar in size; (II) efficiency scores are ascending through the sequence of targets; (III) the target unit at each step is close to the special part of the efficient frontier as much as possible. These properties lead to finding a target that is more achievable in real applications.
\end{abstract}

KeYwords: Data Envelopment Analysis; Target Setting; Returns to Scale; Gradual Improvement.

\footnotetext{
*Department of Computer Sciences, Faculty of Mathematical Sciences and Computer, Kharazmi University, Tehran, Iran, Tel: +98-21-77507772, Fax: +98-21-77630040, Akram.dehnokhalaji@khu.ac.ir

${ }^{\dagger}$ Corresponding author:Department of Mathematics, Faculty of Mathematical Sciences and Computer, Kharazmi University, Tehran, Iran, std_soltani97@khu.ac.ir
} 


\section{Introduction}

Proposed by Charnes et al. (1978), Data Envelopment Analysis (DEA) is a nonparametric technique which is used for evaluating homogenous Decision Making Units (DMUs). The foundation of DEA is based on estimating the so called utility function as the frontier of the Production Possibility Set (PPS) and measuring the distance of each unit from the frontier to define its efficiency score. Some DEA models consider the radial distance (Charnes et al. 1978; Banker et al. 1984) while others are non-radial (Russell 1985; Tone 2001). Different norms can be used in measuring the above-mentioned distance but $l_{1}$-norm is more common since it results in a linear model (Brockett et al. 1997; Pastor et al. 1999). For a single unit, if such distance is positive, it is inefficient. Otherwise, it is said to be a boundary point which can be efficient or inefficient (for more details see Seiford and Thrall (1990)).

One of important problems in recent DEA is how to find a target unit for an inefficient unit to improve its performance. In other words, the Decision Maker (DM) is interested in a target point (efficient projection point on the frontier) that characterizes the inputoutput changes needed for an inefficient unit to become efficient. Conventional DEA models consider this problem, but they yield a target that is usually a far boundary projection point. For instance, the RAM model, proposed by Cooper et al. (1999), determines the furthest target for each inefficient unit. Therefore, several DEA methods are proposed to not only remove this drawback, but also could add some other desirable properties to the selected target. One of the first attempts to find the closest target was done by Frei and Harker (1999). Although the closest target is the unit with the minimum distance from the assessed inefficient unit, but it might be incompatible with facilities of the unit under assessment and can not be achieved in one single step in short term, which is disappointing. For example, in a production plan of factories with the number of employees as an input, there are two ways to remove inefficiencies: increasing outputs or decreasing inputs. It is not reasonable to fire a large number of employees or it is impossible to produce a large amount of products immediately. Therefore, the target unit introduced by one-step benchmarking approaches could be unrealistic and meaningless in practice and one would rather define an admissible distance for variations of inputs and outputs based on the improvement ability of the factory to make it efficient in several steps. Generally, the DM might be interested in using the gradual improvement-based approaches which provide a path of intermediate targets to remove the inefficiency towards reaching the final efficient target. There are different criteria for this gradual improvement that lead to different target paths. For example, Coelli (1998) decreased all inputs that can be improved with the same proportion at each step of 
his proposed method. Lozano and Villa (2005) improved an inefficient unit gradually in a way that the final efficient target is closer to the unit under assessment than the one-step projection point. Estrada et al. (2009) implemented the proximity-based target selection strategy to reach the frontier. Also, intermediate targets proposed by Park et al. (2012) were obtained along some directions based on a similarity criterion. Additionally, Lozano and Calzada-Infante (2017) proposed a new method for gradually improvement of inefficient units in a way that the potential field is ascending through their target path.

In this paper, we propose an algorithm to produce a new path of targets. In our algorithm, we find the intermediate and final targets in a way that they have the same Returns To Scale (RTS) property as the unit under evaluation and the efficiency score of the unit does not get worse at each step of the algorithm. Also, according to the ability of the unit for varying its inputs and outputs, we minimize the ditance of the unit under assessment at each step from a special part of the frontier in order to achieve the final efficient target in fewer steps. In summary, our aim is to construct a finite sequence of targets for each inefficient unit with the following properties: (I) all targets in the sequence and the unit under evaluation belong to the same RTS category; (II) the efficiency scores are ascending in this sequence; (III) the distance between each target and the special part of the frontier is minimized based on the capacity of the unit; (IV) the efficiency score of the final target is equal to one, i.e, the final target is efficient.

The remaining of this paper is organized as follows: Section 2 provides necessary preliminaries to our approach. In section 3 our target setting algorithm is presented. Section 4 illustrates our proposed algorithm with a numerical example and application. Conclusions are finally provided in section 5 .

\section{Preliminaries}

Consider $n$ decision making units $D M U_{j}$, with input vector $x_{j}=\left(x_{1 j}, \ldots, x_{m j}\right)^{t}$ and output vector $y_{j}=\left(y_{1 j}, \ldots, y_{s j}\right)^{t}$, for $j=1, \ldots, n$, where all $x_{i j}$ 's and $y_{r j}$ 's are positive real numbers.

Definition 2.1. The Production Possibility Set (PPS) is defined as:

$$
P=\{(x, y) \mid x \text { can produce } y\}
$$

It is clear that $\mathrm{P}$ can possess different mathematical formulations based on the production technology assumptions (see Cooper et al. 2006), for example $P=\left\{(x, y) \in R^{m+s} \mid x \geq\right.$ 
Table 1: BCC model in envelopment and multiplier forms

Envelopment form:

$\min \theta$

$$
\begin{array}{ll}
\text { s.t. } & \sum_{j=1}^{n} \lambda_{j} x_{j} \leq \theta x_{o}, \\
& \sum_{j=1}^{n} \lambda_{j} y_{j} \geq y_{o}, \\
& \sum_{j=1}^{n} \lambda_{j}=1, \\
& \lambda_{j} \geq 0
\end{array}
$$

Multiplier form:

$$
\begin{aligned}
\max & u y_{o}+u_{0} \\
\text { s.t. } & v x_{o}=1 \\
& u y_{j}-v x_{j}+u_{0} \leq 0 \\
& j=1, \ldots, n \\
& u \geq 0 \\
& v \geq 0
\end{aligned}
$$

$\left.\sum_{j=1}^{n} \lambda_{j} x_{j}, y \leq \sum_{j=1}^{n} \lambda_{j} y_{j}, \lambda \in \Lambda\right\}$ where $\Lambda=\{\lambda \mid \lambda \geq 0\}$ and $\Lambda=\{\lambda \mid 1 \lambda=1, \lambda \geq 0\}$ for Constant RTS (CRS) and Variable RTS (VRS) technologies, respectively. The PPS frontier can be considered as an estimation of the utility function. DEA models measure the distance of units from the PPS frontier in order to determine their efficiency scores (For more details, see Banker et al. 1984; Charnes et al. 1978; Färe and Grosskopf 1985; Seiford and Thrall 1990). In this study the VRS technology is considered and the conventional BCC model is utilizied to measure the efficiency scores. Table 1 represents the BCC model in both envelopment and multiplier forms.

Definition 2.2. $D M U_{o}$ is said to be BCC-efficient if the optimal value of model (2) (or model (3)) is equal to one. Otherwise it is called inefficient.

Definition 2.3. The input-oriented radial projection point of $D M U_{o}$ is defined as $\left(\theta_{o}^{*} x_{o}, y_{o}\right)$ where $\theta_{o}^{*}$ is the optimal value of model (2).

It is obvious that the radial projection point of a given unit lies on the frontier of the PPS. Next theorem provides an important property of model (3) that is useful in what follows.

Theorem 2.1. Assume that $\left(u^{*}, v^{*}, u_{0}^{*}\right)$ is the optimal solution of model (3) evaluating $D M U_{o}$. The hyperplane

$$
H_{o}=\left\{(x, y) \mid u^{*} y-v^{*} x+u_{0}^{*}=0\right\}
$$

is a supporting hyperplane of the PPS at $\left(x_{o}, y_{o}\right)$. Also, each point belonging to $H_{o} \cap P P S$ is BCC-efficient. 
Proof. See Cooper et al. (2006).

For inefficient DMUs, DEA models provide efficient targets to improve their performance. Motivated by Lozano and Villa (2005), we are interested to improve the performance of an inefficient DMU gradually instead of immediately. Lozano and Villa modified the Measure of Inefficiency Proportions (MIP) model (proposed by Cooper et al. (1999)) and instead of reaching the efficient frontier at only one step, they applied the modified MIP model at each step of their algorithm to define intermediate targets. In other words, they presented a sequence of targets to the DM instead of only one target. In continue of their work, we also provide a sequence of targets to the DM. The differences between our proposed approach and the method of Lozano and Villa (2005) are as follow: firstly, the returns to scale will not change during our algorithm, secondly, our proposed target at each step is the closest one to the frontier. Returns to Scale (RTS) is an important property of the production points in economics. The RTS of a production point relates to the rate of changes in outputs when the inputs are increased proportionally. In a production process, a DMU exhibits an increasing (decreasing) RTS if the proportionate increase in outputs is larger (smaller) than the proportionate increase in inputs and a constant RTS occurs for a unit if the proportionate increase in outputs is equal to the proportionate increase in inputs. In this regard, DMUs can be classified into three categories: IRS, DRS and CRS. We can address two more terms which are well-known in DEA literature: Non-increasing RTS (NIRS) which includes both decreasing and constant RTS cases and Non-decreasing RTS (NDRS) which includes both increasing and constant RTS cases.

In what follows we present the mathematical formulation of a subset of the PPS with the points belonging to the same RTS category.

\subsection{A subset of the PPS consisting of production points with the same RTS property}

Suppose that $D M U_{o}$ is a unit under evaluation and $\left(u^{*}, v^{*}, u_{0}^{*}\right)$ is an optimal solution for model (3) and $\theta_{o}^{*}$ is the optimal value of objective function of model (2) evaluating $D M U_{o}$. Let $\left(\hat{X}_{o}, \hat{Y}_{o}\right)=\left(\theta_{o}^{*} x_{o}, y_{o}\right)$ be the radial projection point. Nasrabadi et al. (2014) specified a subset of the PPS consisting of all units whose radial projection points belong to the same supporting hyperplane. In other words, they characterized the mathematical formulation of set

$$
P_{H_{o}}=\left\{(\bar{x}, \bar{y}) \in \operatorname{PPS} \mid\left(\bar{\theta}^{*} \bar{x}, \bar{y}\right) \in H_{o}\right\}
$$


in which, $\bar{\theta}^{*}$ is the optimal solution of model $(2)$ evaluating $(\bar{x}, \bar{y})$. The region $P_{H_{o}}$ is shown in Figure (1) for the single-input single-output case.

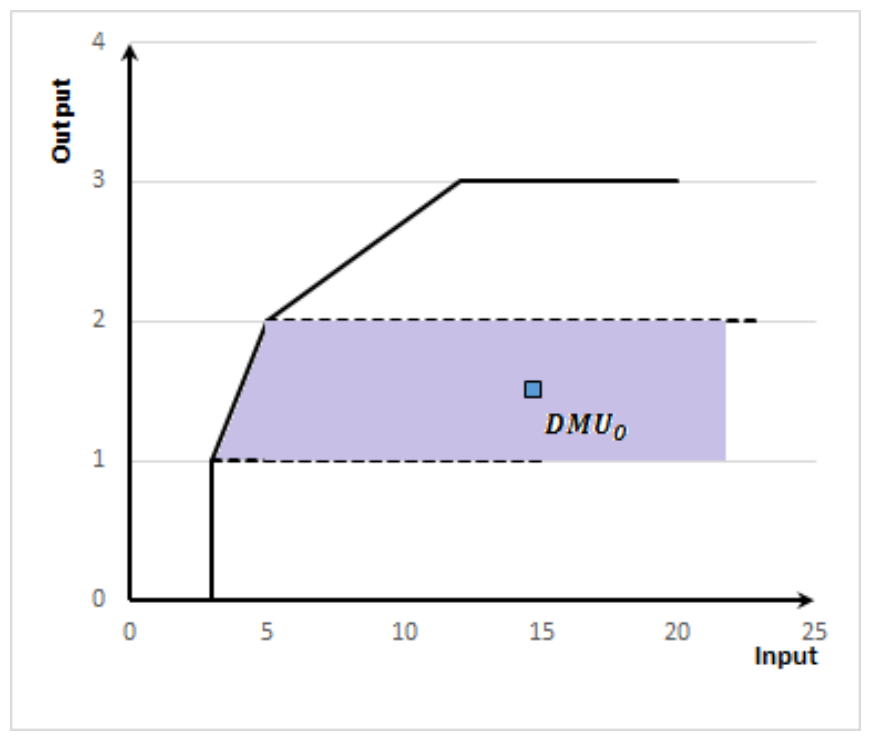

Figure 1: $P_{H_{o}}$ in one-input and one-output case

Suppose that $\left\{i_{1}, \ldots, i_{q}\right\}=\left\{i \mid v_{i}^{*}=0\right\}$ and $\left\{r_{1}, \ldots, r_{p}\right\}=\left\{r \mid u_{r}^{*}=0\right\}$. Also, let

$$
K_{H_{o}}=\left\{\left(x_{j}, y_{j}\right) \mid j \in\{1, \ldots, n\},\left(x_{j}, y_{j}\right) \in H_{o}\right\}
$$

For an arbitrary finite set $A=\left\{a_{1}, \ldots, a_{n}\right\}$, the convex hull of $A$ is defined as:

$$
\operatorname{Conv}(A)=\left\{\sum_{j=1}^{n} \lambda_{j} a_{j} \mid \sum_{j=1}^{n} \lambda_{j}=1, \lambda_{j} \geq 0, j=1, \ldots, n\right\}
$$

With these notations, Nasrabadi et al. (2014) proved that

$$
\begin{aligned}
& P_{H_{o}}=\left\{\left(\alpha x+\sum_{k=1}^{q} d_{k} e_{i_{k}}, y-\sum_{l=1}^{p} d_{l}^{\prime} e_{r_{l}}^{\prime}\right) \mid \alpha \geq 1,(x, y) \in \operatorname{Conv}\left(K_{H_{o}}\right),\right. \\
& \left.\left(d, d^{\prime}\right)=\left(d_{1}, \ldots, d_{q}, d_{1}^{\prime}, \ldots, d_{p}^{\prime}\right) \geq 0,\left(d_{1}^{\prime}, \ldots, d_{p}^{\prime}\right) \leq\left(y_{r_{1} o}, \ldots, y_{r_{p} o}\right)\right\}
\end{aligned}
$$

where $e_{i_{k}}$ is the unit vector in $R^{m}$ with the $i_{k}{ }^{\text {th }}$ component equal to 1 and $e_{r_{l}}^{\prime}$ is the unit vector in $R^{s}$ with the $r_{l}^{\text {th }}$ component equal to 1 . They also proved the following basic theorem:

Theorem 2.2. Assuming $\left(u^{*}, v^{*}, u_{0}^{*}\right)$ as an optimal solution of model (3) when assessing $D M U_{o}$, we have:

(i) If $u_{0}^{*}=0$, then Constant RTS prevails at any $(\bar{X}, \bar{Y}) \in P_{H_{o}}$. 
(ii) If $u_{0}^{*}>0$, then Non-Decreasing RTS prevails at any $(\bar{X}, \bar{Y}) \in P_{H_{o}}$.

(iii) If $u_{0}^{*}<0$, then Non-Increasing RTS prevails at any $(\bar{X}, \bar{Y}) \in P_{H_{o}}$.

Proof. See Nasrabadi et al. (2014).

From Theorem 2.2, we can conclude that if $D M U_{o}$ belongs to the Constant RTS (CRS) category, then all point in $P_{H_{o}}$ belong to the CRS category as well. Similar statements hold true for NIRS and NDRS categories. Therefore, all points in region $P_{H_{o}}$ are the most similar units to $D M U_{o}$ in the PPS regarding the RTS property.

In step-wise benchmarking literature, a key point in target setting and performance improvement for inefficient units is the similarity criterion. Different similarity criteria lead to different selections of targets. Hence, the way the performance of a unit is improved depends on the similarity criterion. In this paper, we define the similarity criterion based on the RTS property and efficiency score. In other words, we move an inefficient unit towards the efficient frontier through a sequence of targets all belonging to the same RTS category as the unit under evaluation and their efficiency scores are ascending. Also, in order to reach the frontier in fewer steps, we minimize the distance of the unit under assessment at each step from the efficienct frontier. In this way, we project an inefficient unit on a production point with a similar size gradually which is more meaningful in practice.

Hence, according to the similarity criterion and considering Theorem 2.2, we have to move in a specific part of region $P_{H_{o}}$ in order to find a sequence of targets with the same RTS. Also, another criterion is to improve the efficiency score, i.e., to remain in the region with better efficiency scores than the unit under assessment. Thus, in the next section, we firstly characterize the mathematical formulation of this region and then we present an algorithm to find the path of targets for each inefficient unit.

\section{The Proposed Target Setting Algorithm}

In this section we present our proposed method that its aim is improving an inefficient unit gradually which is consistent with the capacity of the unit under assessment. Compared with Lozano and Villa (2005), we also present a sequence of targets that their efficiency scores are ascending in this sequence and it ends with an efficient target. Our proposed method has two more benefits. Firstly, returns to scale does not change during the improvement path and it is the same as the unit under assessment. Secondly, the proposed intermediate target 
at each step is the nearest one to the special part of the frontier with the same returns to scale as the unit under assessment.

Let $D M U_{o}$ be an inefficient unit. We search in a specific part of region $P_{H_{o}}$ to find the most similar target. We build up an iterative procedure to find a path of targets converging to an efficient projection point. All targets in this path belong to region $P_{H_{o}}$ and they belong to the same RTS category, consequently. In order to find the sequence of similar targets for $D M U_{o}$, we also need a subset of the PPS consisting of the units with the efficiency score greater than or equal to the efficiency score of the unit under evaluation and we limit our search to the intersection of this subset and $P_{H_{o}}$. In this way, we can guarantee that RTS does not change during the algorithm and the efficiency scores of targets are ascending in the sequence. Note that our algorithm terminates when an efficient target is obtained. The next two theorems provide what we need.

Theorem 3.1. Let $(x, y) \in \bar{H}_{o}=\left\{(x, y) \in P P S \mid u^{*} y-\theta_{o}^{*} v^{*} x+u_{0}^{*} \geq 0\right\}$ and $\bar{\theta}$ be the efficiency score of $(x, y)$. Then $\bar{\theta} \geq \theta_{o}^{*}$.

Proof. Suppose that $(x, y) \in \bar{H}_{o}$. Therefore, $u^{*} y-\theta_{o}^{*} v^{*} x+u_{0}^{*} \geq 0$ and there exists $s \geq 0$ such that

$$
u^{*} y-\theta_{o}^{*} v^{*} x+u_{0}^{*}-s=0
$$

Since $v^{*} x>0$, there exists $\eta \geq 0$ such that $s=\eta v^{*} x$. Now we rewrite the above equation as follows:

$$
u^{*} y-\theta_{o}^{*} v^{*} x+u_{0}^{*}-\eta v^{*} x=0 \Rightarrow u^{*} y-\left(\theta_{o}^{*}+\eta\right) v^{*} x+u_{0}^{*}=0,
$$

implying that $\left(\left(\theta_{o}^{*}+\eta\right) x, y\right) \in H_{o}$. Since $\left(\left(\theta_{o}^{*}+\eta\right) x, y\right)$ is either on the frontier of the PPS or out of it, evaluating it by model (2) results in an optimal value greater than or equal to 1. On the other hand, let $\bar{\theta}$ be the optimal value of model $(2)$ evaluating $(x, y)$. Then $\frac{\bar{\theta}}{\theta_{o}^{*}+\eta}$ is the optimal value of model $(2)$ evaluating $\left(\left(\theta_{o}^{*}+\eta\right) x, y\right)$. Therefore, $\frac{\bar{\theta}}{\theta_{o}^{*}+\eta} \geq 1$ and consequently $\bar{\theta} \geq \theta_{o}^{*}+\eta \geq \theta_{o}^{*}$. 


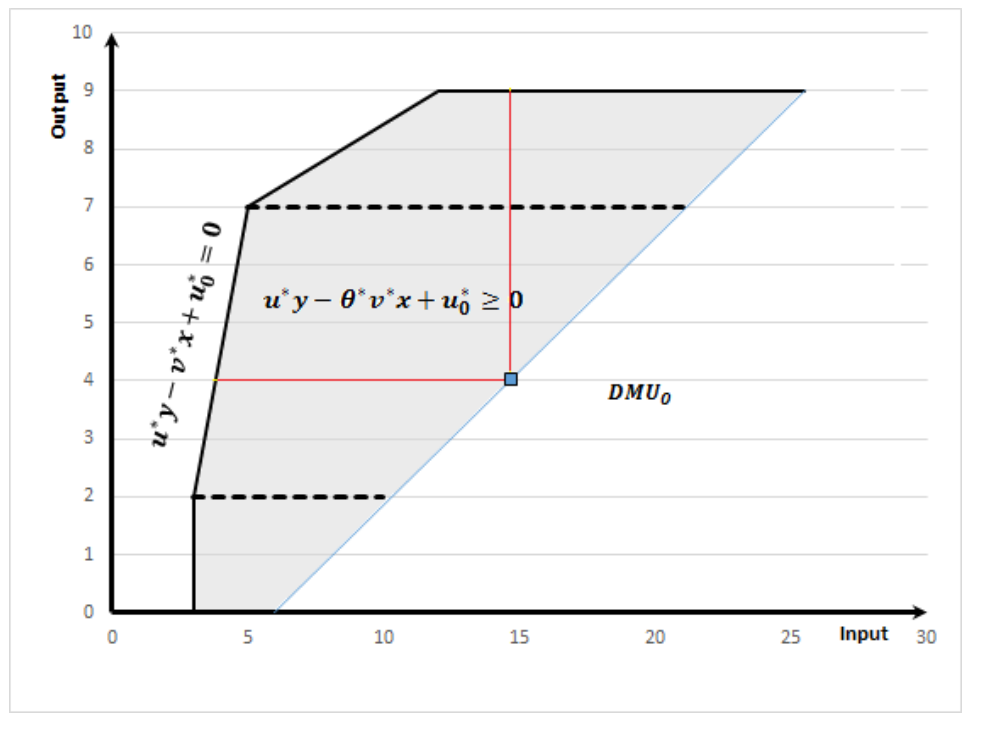

Figure 2: Illustration of Theorem 3.1 in a single-input single-output case

Consider a PPS in case of single-input single-output with the VRS assumption. An illustration of Theorem 3.1 is provided in Figure 2. Here $\bar{H}_{o}$ is the shaded region. As can be seen, the subset of the PPS consisting of the points dominating $D M U_{o}$ is also a subset of $\bar{H}_{o}$. This inclusion holds true in general.

Theorem 3.2. Let $(x, y) \in P P S$ and $y \geq y_{o}, x \leq x_{o}$. Then $(x, y) \in \bar{H}_{o}$.

Proof. Since $y \geq y_{o}, x \leq x_{o}$ and $u^{*}, v^{*}$ and $\theta_{o}^{*}$ are nonnegative, we have $u^{*} y-\theta_{o}^{*} v^{*} x+u_{0}^{*} \geq$ $u^{*} y_{o}-\theta_{o}^{*} v^{*} x_{o}+u_{0}^{*}$. Also, according to the equation $v^{*} x_{o}=1$, we have $u^{*} y_{o}-\theta_{o}^{*} v^{*} x_{o}+u_{0}^{*}=$ $u^{*} y_{o}-\theta_{o}^{*}+u_{0}^{*}=0$. The latter equality holds true because $u^{*} y_{o}+u_{0}^{*}=\theta_{o}^{*}$ by the strong duality theorem. Thus, $u^{*} y-\theta_{o}^{*} v^{*} x+u_{0}^{*} \geq 0$ and consequently $(x, y) \in \bar{H}_{o}$.

Based on what mentioned above, we solve model (3) to determine inefficient units and their corresponding supporting hyperplanes. Then we solve the following model to find the most similar target to $D M U_{o}$. 


$$
\begin{array}{cl}
\min & \operatorname{dist}((x, y),(\bar{x}, \bar{y})) \\
\text { s.t. } & (x, y) \in P_{H_{o}} \\
& (x, y) \in \bar{H}_{o} \\
& u^{*} \bar{y}-v^{*} \bar{x}+u_{0}^{*}=0
\end{array}
$$

where $(x, y)$ belongs to the admisible region for variation of inputs and outputs of $D M U_{o}$ and $(\bar{x}, \bar{y})$ is an arbitrary point on the corresponding supporting hyperplane. Let $\left(x^{*}, y^{*}\right)$ be the target point obtained from solving model (8). If $\left(x^{*}, y^{*}\right)$ is efficient, we identify it as the final projection point and stop our algorithm. Otherwise, we set $\left(x_{o}, y_{o}\right)=\left(x^{*}, y^{*}\right)$ and repeat the above procedure.

To illustrate the algorithm, consider a single-input single-output case depicted in Figure 3. The RTS category of all points in the shaded area is the same as $D M U_{A}$ category. Such points have the efficiency scores greater than or equal to $\theta_{A}^{*}$ as well. The rectangular area in this figure depicts the admissible region for inputs and outputs changes of $D M U_{A}$. Target $A_{1}$ is obtained after solving model (8) once. Since $A_{1}$ is inefficient, we solve model (8) for $A_{1}$ and find the next target $A_{2}$. We repeat this procedure until we reach the efficient frontier where the final projection point is located. One can find the sequence of obtained targets in Figure 3 .

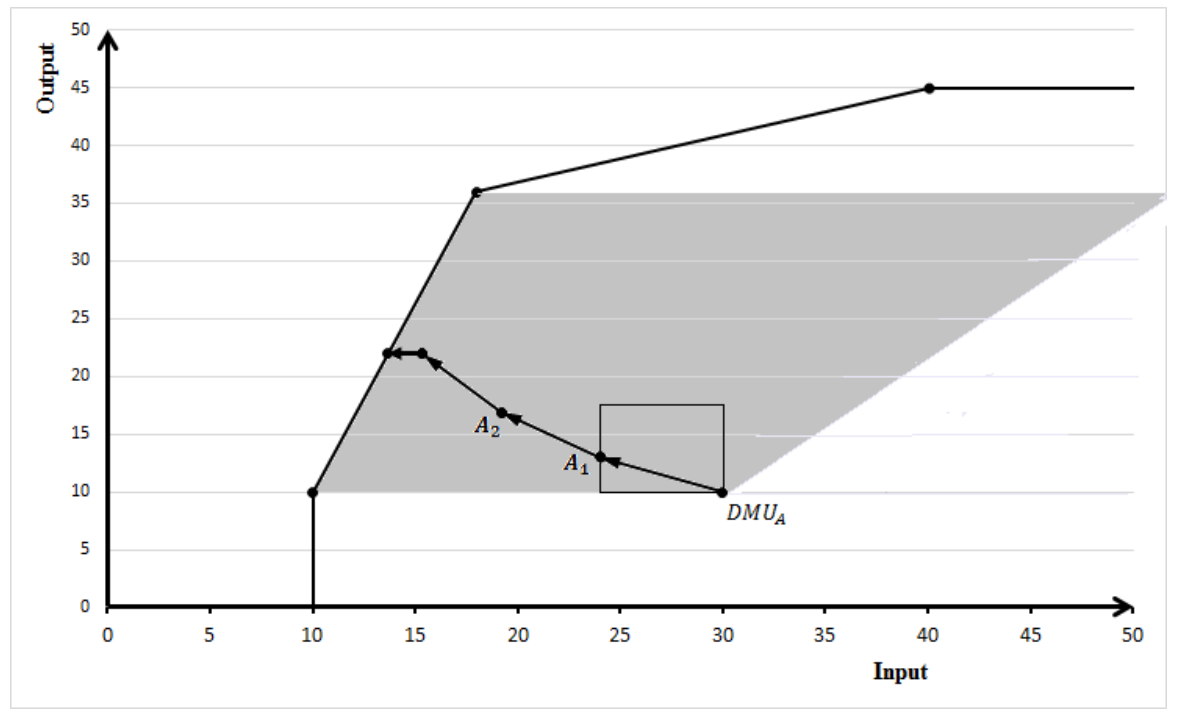

Figure 3: Illustration of the target setting procedure 
Substituting the mathematical formulation of $P_{H_{o}}$ in model (8) yields nonlinear constraints. Below, we present a lemma that is necessary to prove Theorem 3.3 in order to provide an equivalent formula for $P_{H_{o}}$ resulting in a linear target setting model.

Lemma 3.1. Region $P_{H_{o}}$ is equal to

$$
\begin{aligned}
\hat{H}_{o}=\left\{(x, y) \mid x=\bar{x}+\sum_{k=1}^{q} d_{k} e_{i_{k}}, y=\bar{y}-\sum_{l=1}^{p} d_{l}^{\prime} e_{r_{l}}^{\prime}\right), \\
\left(\begin{array}{c}
\bar{x} \\
\bar{y}
\end{array}\right)=\sum_{j \in K_{H_{o}}} \lambda_{j}\left(\begin{array}{c}
\alpha_{j} x_{j} \\
y_{j}
\end{array}\right), \alpha_{j} \geq 1, \sum_{j \in K_{H_{o}}} \lambda_{j}=1, \lambda_{j} \geq 0, \\
\left.\left(d, d^{\prime}\right)=\left(d_{1}, \ldots, d_{q}, d_{1}{ }^{\prime}, \ldots, d_{p}{ }^{\prime}\right) \geq 0,\left(d_{1}{ }^{\prime}, \ldots, d_{p}{ }^{\prime}\right) \leq\left(y_{r_{1} o}, \ldots, y_{r_{p} o}\right)\right\} .
\end{aligned}
$$

Proof. If $\alpha_{j}=\alpha$ for all $j$, then $P_{H_{o}} \subset \hat{H}_{o}$. Conversely, if we set $\alpha=\frac{\sum_{j \in K_{H_{o}}} \lambda_{j} \alpha_{j} x_{j}}{\sum_{j \in K_{H_{o}}} \lambda_{j} x_{j}}$, then we can conclude that $\hat{H}_{o} \subset P_{H_{o}}$. Thus $\hat{H}_{o}=P_{H_{o}}$

Theorem 3.3. The two sets $\hat{H}_{o}$ and $\tilde{H}_{o}$ are equal where

$$
\begin{gathered}
\tilde{H}_{o}=\left\{(x, y) \mid x \geq \sum_{j \in K_{H_{o}}} \lambda_{j} x_{j}, y=\sum_{j \in K_{H_{o}}} \lambda_{j} y_{j}-\sum_{l=1}^{p} d_{l}{ }^{\prime} e_{r_{l}}{ }^{\prime} \sum_{j \in K_{H_{o}}} \lambda_{j}=1, \lambda_{j} \geq 0,\right. \\
\left.d^{\prime}=\left(d_{1}{ }^{\prime}, \ldots, d_{p}{ }^{\prime}\right) \geq 0,\left(d_{1}{ }^{\prime}, \ldots, d_{p}{ }^{\prime}\right) \leq\left(y_{r_{1} o}, \ldots, y_{r_{p} o}\right)\right\} .
\end{gathered}
$$

Proof. It is clear that $\hat{H}_{o} \subset \tilde{H}_{o}$. To prove $\tilde{H}_{o} \subset \hat{H}_{o}$, let $(x, y) \in \tilde{H}_{o}$. Hence, $x \geq \sum_{j \in K_{H_{o}}} \lambda_{j} x_{j}$. Therefore, there exists $S \geq 0$ such that

$$
\begin{aligned}
x & =\sum_{j \in K_{H_{o}}} \lambda_{j} x_{j}+S \quad=\sum_{j \in K_{H_{o}}} \lambda_{j} x_{j}+\sum_{j \in K_{H_{o}}} \eta_{j}\left(\lambda_{j} x_{j}\right) \\
& =\sum_{j \in K_{H_{o}}}\left(1+\eta_{j}\right)\left(\lambda_{j} x_{j}\right)=\sum_{j \in K_{H_{o}}} \alpha_{j} \lambda_{j} x_{j},
\end{aligned}
$$

where $\eta_{j} \geq 0$ and $\alpha_{j}=1+\eta_{j}$ for $j \in K_{H_{o}}$. Hence, $(x, y) \in \hat{H}_{o}$. Thus, $\tilde{H}_{o}=\hat{H}_{o}$.

We use the following notations for more simplification in what follows: 
$o: \quad$ index of the unit under evaluation.

$L_{I}^{t}$ : lower bounds of admissible changes of inputs at step $t$.

$U_{O}^{t}: \quad$ upper bounds of admissible changes of outputs at step $t$.

$t: \quad$ index of the target at step $t$.

$x^{t}, y^{t}$ : input and output vectors of the intermediate target at step $t$

$\left(x^{0}=x_{o}, y^{0}=y_{o}\right)$

$\theta^{* t}$ : the optimal value of model (2) evaluating $\left(x^{t}, y^{t}\right)$.

$\left(\theta^{* 0}=\theta_{o}^{*}\right)$

Based on the above theory, we can substitute $P_{H_{o}}$ with $\tilde{H}_{o}$ and rewrite model (8) as follows:

$$
\begin{array}{ll}
\min \quad & 1 \frac{|x-\bar{x}|}{x_{o}}+1 \frac{|y-\bar{y}|}{y_{o}} \\
\text { s.t. } \quad & \sum_{j \in k_{H_{o}}} \lambda_{j}^{t} x_{j} \leq x \\
& \sum_{j \in k_{H_{o}}} \lambda_{j}^{t} y_{j}-\sum_{l=1}^{p} d_{l}^{t} e_{r_{l}}=y \\
& \sum_{j \in k_{H_{o}}} \lambda_{j}^{t}=1 \\
& u_{o}^{*} y-\theta^{*^{t-1}} v_{o}^{*} x+u_{0}^{*} \geq 0 \\
& u_{o}^{*} \bar{y}-v_{o}^{*} \bar{x}+u_{0}^{*}=0 \\
& L_{I}^{t} \leq \frac{x-x^{t-1}}{x^{t-1}} \leq 0 \\
& 0 \leq \frac{y-y^{t-1}}{y^{t-1}} \leq U_{O}^{t} \\
& \left(d_{1}^{t}, \ldots, d_{p}^{t}\right) \leq\left(y_{r_{1} o}, \ldots, y_{r_{p} o}\right),
\end{array}
$$

in which all variables are positive and 1 is a vector with all components equal to 1 . The constraints (9a)-(9c) are corresponding to the constraint (8a) and the constraint (9d) is obtained from the constraint (8b) of model (8). The constraint (9e) is the same with the constraint (8c) and (9f) and (9g) characterize the admissible region for variation of inputs 
and outputs. Note that, lower and upper bounds are parameters that have to be determined by the DM according to the ability of $D M U_{o}$ to change its inputs and outputs. These bounds can be changed at each step or remain fixed during the algorithm.

Although the objective function of model (9) is nonlinear, it can be easily linearized by the change of variables as $x-\bar{x}=a-b, y-\bar{y}=c-d ; a, b, c, d \geq 0$ and equalities $|x-\bar{x}|=a+b,|y-\bar{y}|=c+d$.

After solving model (9), the intermediate target at step $t$ is as follows:

$$
\begin{aligned}
& x^{t}=x^{*} \\
& y^{t}=y^{*}
\end{aligned}
$$

where subscript "*" stands for the optimality in model (9). In what follows, we show that model (9) is feasible and possesses a finite optimal solution.

Theorem 3.4. Model (9) is feasible and has a finite optimal solution.

Proof. Since $\left(x_{o}, y_{o}\right) \in P_{H_{o}}$ and if $\left(x_{t-}, y_{t-1}\right) \in P_{H_{o}}$ then $\left(x_{t}, y_{t}\right) \in P_{H_{o}}$, so $P_{H_{o}} \neq \emptyset$ at each step. As a result, there are cofficients $\lambda_{j}^{t}$ and $d_{l}^{t}$ that lead to the feasibility of model (9) for the selection of other variables as follows:

$$
(x, y)=\left(x_{t-1}, y_{t-1}\right), \quad(\bar{x}, \bar{y})=\left(\theta^{* t-1} x_{t-1}, y_{t-1}\right)
$$

Hence, model (9) is feasible. Also, the existence of a lower bound for the objective function guarantees that the optimal value is finite.

Now, we present our algorithm to determine a sequence of targets for inefficient unit $D M U_{o}$.

\section{TARGET SETTING ALGORITHM}

Step1: Set $t=0$

Step2: Set $x^{0}=x_{o}, y^{0}=y_{0}$.

Step3: Evaluate $\left(x_{o}, y_{o}\right)$ by model (3) and determine values $u_{o}^{*}, v_{o}^{*}, u_{0}^{*}$.

Step4: Evaluate $\left(x^{t}, y^{t}\right)$ by model $(2)$ and determine the value $\theta^{t^{*}}$. 
Step5: If $\theta^{t^{*}}=1$, stop. If $\theta^{t^{*}}<1$, Go to step 6 .

Step6: Set $t \leftarrow t+1$ and solve model (9).

Step7: Set $\left(x^{t}, y^{t}\right)=\left(x^{*}, y^{*}\right)$ and go to step 4 .

Since we move in region $P_{H_{o}}$ and according to the theorem (2.2), it is easy to understand that returns to scale does not change during our algorithm for $D M U_{o}$. Also, since $\theta^{t^{*}}$ is increasing and $\theta^{t^{*}} \leq 1$, the algorithm terminates after finite several steps. Note that the final target is efficient according to the termination criterion. In the next section, we illustrate our proposed target setting algorithm by a numerical example first, and then we present an empirical example to show its performance.

\section{Numerical results for the target setting algorithm}

Consider 6 units with one input and one output. The data is taken from Lozano and Villa (2005) and is reported in Table 2. The Production Possibility Set for this example is shown in Figure 4.

Table 2: Data for the numerical example taken from Lozano and Villa (2005)

\begin{tabular}{cccccc}
\hline & & \multicolumn{4}{c}{ Results of the conventional BCC model } \\
\hline$D M U$ & $\mathrm{x}$ & $\mathrm{y}$ & $\theta^{*}$ & $x^{*}$ & $y^{*}$ \\
\hline$A$ & 10 & 10 & 1.00 & 10 & 10 \\
$B$ & 18 & 36 & 1.00 & 18 & 36 \\
$C$ & 30 & 10 & 0.33 & 10 & 10 \\
$D$ & 35 & 40 & 0.69 & 24.17 & 40 \\
$E$ & 55 & 60 & 1.00 & 55 & 60 \\
$F$ & 60 & 20 & 0.22 & 13.08 & 20 \\
\hline
\end{tabular}

It can be seen that units $A, B$ and $E$ are efficient and are their own targets but units $C$, $D$ and $F$ are inefficient. Assume that $L_{I}=-0.2$ and $U_{O}=0.3$. If we apply the conventional DEA models to improve the performance of inefficient units, then we have to reach the frontier at only one step. The BCC-efficiency scores of these units and their targets are reported in the last three column of Table 2. Consider $D M U_{F}$, with the activity vector $(60,20)$ and BCC-efficiency score equal to 0.22 . The DM must reduce about $78 \%$ of the input of $D M U_{F}$ at a single step to make it efficient, while the capacity for the input reduction is considered at most 20\%. Therefore, it is impossible for the DM to reach this target immediately. Hence, it is reasonable to improve the unit gradually through a sequence of targets. In Continue, 


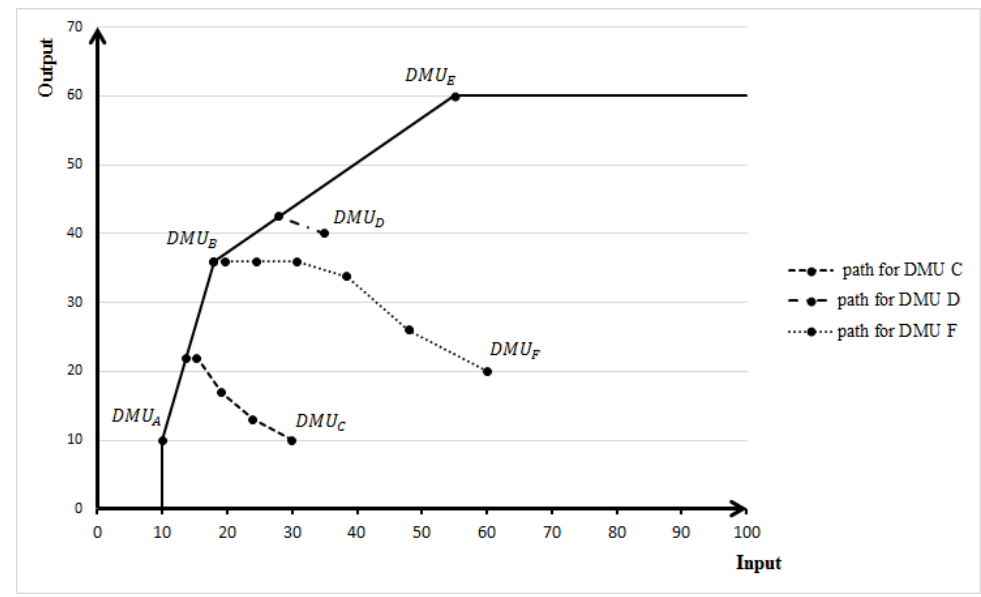

Figure 4: The sequence of targets for inefficient units for the numerical example

we illustrate how our target setting algorithm performs, applying it on unit $F$, which has a non-decreasing returns to scale.

If we apply the proposed algorithm on unit $F$, then the first intermediate target activity vector is equal to $(48,26)$. So, at the first step, it is sufficient to reduce the input by 12 units and increase the output by 6 units, respectively. With this improvement, the efficiency score will be 0.31 and the returns to scale remains non-decreasing. We repeat the target setting algorithm six times to find the final efficient target $(18,36)$ with the same RTS as $D M U_{F}$. The results of this example and the path of intermediate targets for each inefficient unit are shown in Table 3 and Figure 4, respectively. Note that, for each inefficient DMU, the RTS category does not change during the algorithm and efficiency scores are ascending as we expected.

\subsection{Empirical example}

Consider 26 geographical districts that constitute Rio de Janerio municipality in Brazil with two inputs and one output where the number of dentists and potential target demands are inputs and the total number of preventive plus conclusive dental procedures is the output for each unit. The data extracted from Lins et al. (2004) and the BCC efficiency scores for all units are reported in Table 4. 
Table 3: The target setting algorithm results for the numerical example

\begin{tabular}{|c|c|c|c|c|c|c|c|c|c|}
\hline & & Step0 & Step1 & Step2 & Step3 & Step4 & Step5 & Step6 & \\
\hline \multirow[t]{4}{*}{$D M U_{A}$} & $\mathrm{x}$ & 10 & & & & & & & \\
\hline & $\mathrm{y}$ & 10 & & & & & & & \\
\hline & BCC Eff.score & 1.00 & & & & & & & \\
\hline & $u_{0}^{*}$ & 0.69 & $(\mathrm{NDR})^{1}$ & & & & & & \\
\hline \multirow[t]{4}{*}{$\overline{D M U_{B}}$} & $\mathrm{x}$ & 18 & & & & & & & \\
\hline & $\mathrm{y}$ & 36 & & & & & & & \\
\hline & BCC Eff.score & 1.00 & & & & & & & \\
\hline & $u_{0}^{*}$ & 0.38 & (NDR) & & & & & & \\
\hline \multirow[t]{4}{*}{$D M U_{C}$} & $\mathrm{x}$ & 30 & 24 & 19.20 & 15.36 & 13.68 & & & \\
\hline & $\mathrm{y}$ & 10 & 13 & 16.90 & 21.97 & 21.97 & & & \\
\hline & BCC Eff.score & 0.33 & 0.46 & 0.63 & 0.89 & 1.00 & & & \\
\hline & $u_{0}^{*}$ & 0.23 & 0.29 & 0.36 & 0.45 & 0.44 & (NDR) & & \\
\hline \multirow[t]{4}{*}{$D M U_{D}$} & $\mathrm{x}$ & 35 & 28 & & & & & & \\
\hline & $\mathrm{y}$ & 40 & 42.49 & & & & & & \\
\hline & BCC Eff.score & 0.69 & 1.00 & & & & & & \\
\hline & $u_{0}^{*}$ & -1.07 & -1.34 & $(\mathrm{NIR})^{2}$ & & & & & \\
\hline \multirow[t]{4}{*}{$D M U_{E}$} & $\mathrm{x}$ & 55 & & & & & & & \\
\hline & $\mathrm{y}$ & 60 & & & & & & & \\
\hline & BCC eff.score & 1.00 & & & & & & & \\
\hline & $u_{0}^{*}$ & -0.68 & (NIR) & & & & & & \\
\hline \multirow[t]{4}{*}{$D M U_{F}$} & $\mathrm{x}$ & 60 & 48 & 38.4 & 30.72 & 24.58 & 19.66 & 18 & \\
\hline & $\mathrm{y}$ & 20 & 26 & 33.80 & 36 & 36 & 36 & 36 & \\
\hline & BCC Eff.score & 0.22 & 0.31 & 0.45 & 0.59 & 0.73 & 0.92 & 1.00 & \\
\hline & $u_{0}^{*}$ & 0.11 & 0.14 & 0.18 & 0.22 & 0.28 & 0.35 & 0.38 & (NDR) \\
\hline
\end{tabular}

$1\left(u_{0}^{*}>0\right.$ means Non-Decreasing Returns to Scale)

${ }^{2}\left(u_{0}^{*}<0\right.$ means Non-Increasing Returns to Scale) 
Table 4: Data for the empirical example taken from Lins et al. (2004)

\begin{tabular}{|c|c|c|c|c|}
\hline \multirow{2}{*}{$\begin{array}{l}\text { DMUs: } \\
\text { Districts }\end{array}$} & \multicolumn{2}{|l|}{ Inputs: } & \multirow{2}{*}{$\begin{array}{l}\text { Outputs: } \\
\text { Preventive plus } \\
\text { conclusive procedures }\end{array}$} & \multirow[b]{2}{*}{$\begin{array}{l}\text { BBC-efficiency } \\
\text { score }\end{array}$} \\
\hline & Number of dentists & Target population & & \\
\hline 1 Portuaria & 6 & 5720 & 22105 & 0.99 \\
\hline 2 Centro & 21 & 2053 & 26884 & 0.88 \\
\hline 3 Rio Comprido & 17 & 7529 & 66836 & 1.00 \\
\hline 4 Botafogo & 25 & 5667 & 83629 & 1.00 \\
\hline 5 Copacabana & 7 & 2612 & 22477 & 1.00 \\
\hline 6 Lagoa & 31 & 8892 & 44985 & 0.43 \\
\hline 7 Sao Cristovao & 12 & 8604 & 42101 & 0.79 \\
\hline 8 Tijuca & 13 & 5705 & 35818 & 0.74 \\
\hline 9 Vila Isabel & 38 & 7023 & 91465 & 1.00 \\
\hline 10 Ramos & 22 & 50726 & 34340 & 0.33 \\
\hline 11 Penha & 19 & 34172 & 58424 & 0.57 \\
\hline 12 Inhauma & 21 & 13320 & 63294 & 0.69 \\
\hline 13 Meier & 50 & 25721 & 103245 & 0.50 \\
\hline 14 Iraja & 20 & 16652 & 71818 & 0.75 \\
\hline 15 Madureira & 25 & 35332 & 92136 & 0.68 \\
\hline 16 Jacarepague & 46 & 42185 & 101144 & 0.43 \\
\hline 17 Bangu & 44 & 81238 & 170336 & 0.70 \\
\hline 18 Campo Grande & 39 & 56396 & 146758 & 0.69 \\
\hline 19 Santa Cruz & 46 & 49542 & 253913 & 1.00 \\
\hline 20 Ilha do Governador & 32 & 13973 & 122375 & 1.00 \\
\hline 21 Paqueta & 5 & 205 & 3507 & 1.00 \\
\hline 22 Anchieta & 10 & 19521 & 47231 & 0.91 \\
\hline 23 Santa Tereza & 6 & 3493 & 23416 & 1.00 \\
\hline 24 Barra da Tijuca & 21 & 6293 & 30488 & 0.46 \\
\hline 25 Pavuna & 8 & 30258 & 42655 & 1.00 \\
\hline 26 Guaratiba & 11 & 15181 & 59033 & 1.00 \\
\hline
\end{tabular}

We apply our proposed target setting algorithm to find intermediate targets for inefficient units to improve them gradually. The number of intermediate targets is different for each unit and not only depends on their inefficiency scores but also on lower and upper bounds of admissible changes of inputs and outputs at each step. We assume that all inputs can be decreased and all outputs can be increased by 10\%. These ranges can be changed and selected for each input and output, separately. It is obvious that the DM can reach the final 
projection point in fewer steps by increasing the lower and upper bounds if it is possible for the organization. In this example, the number of intermediate targets varies between 1 and 8. For instance, the intermediate targets along with their $u_{0}^{*}$ values for $D M U_{12}$ are reported in Table 5. As can be seen from Table 5, all intermediate targets have the same returns to scale as $D M U_{12}$. For this unit to become efficient gradually, the DM can reallocate two dentists in other districts and increase the production from 63294 to 69623.40 to reach the first target with the efficiency score of 0.82 . The second target is characterized by a reduction in the number of dentists from 19 to 18 and an increase in the production. In this step, the efficiency score increases to 0.92. The final efficient target is achieved with the reduction in the number of dentists and no change in the target population and production. All targets and the unit under evaluation belong to NDR category, as we expected.

Table 5: Intermediate targets for $D M U_{12}$.

\begin{tabular}{|c|c|c|c|c|c|}
\hline \multicolumn{2}{|l|}{ Variables } & Number of dentists & Target population & Output & $u_{0}^{*}$ \\
\hline Observed DMU & (12)Inhauma & 21 & 13320 & 63294 & 0.034 \\
\hline \multirow[t]{3}{*}{ Targets } & 1 & 18.90 & 13320 & 69623.40 & 0.036 \\
\hline & 2 & 17.01 & 13320 & 76585.74 & 0.039 \\
\hline & 3 & 15.82 & 13320 & 76585.74 & 0.041 \\
\hline
\end{tabular}

Table 6 reports the number of intermediate targets and the final projection point for each decision making unit along with the $u_{0}^{*}$ value for the final target. As we expected, the RTS category does not change during the algorithm and our algorithm stops in finite steps with a BCC-efficient unit as the final target. Note that, the target point for each efficient unit is itself. 
Table 6: Results for the empirical example

\begin{tabular}{|c|c|c|c|c|c|c|}
\hline \multirow[t]{2}{*}{ DMUs } & \multirow[t]{2}{*}{ Step } & \multicolumn{2}{|l|}{ Inputs } & \multirow{2}{*}{$\begin{array}{l}\text { Output } \\
\text { Preventive plus } \\
\text { conclusive procedure }\end{array}$} & \multirow[t]{2}{*}{$u_{0}^{*}$} & \multirow[t]{2}{*}{ No. of steps } \\
\hline & & $\begin{array}{l}\text { Number of } \\
\text { dentists }\end{array}$ & $\begin{array}{l}\text { Target } \\
\text { poulation }\end{array}$ & & & \\
\hline \multirow[t]{2}{*}{1} & Step0 & 6 & 5720 & 22105 & 0.804 & 1 \\
\hline & Final Step & 5.93 & 5720 & 22105 & 0.813 & \\
\hline \multirow[t]{2}{*}{2} & Step0 & 21 & 2053 & 26884 & -0.017 & 2 \\
\hline & Final Step & 21 & 2053 & 30615.29 & -0.017 & \\
\hline \multirow[t]{2}{*}{6} & Step0 & 31 & 8892 & 44985 & 0.060 & 8 \\
\hline & Final Step & 20.34 & 8892 & 80049.64 & 0.000 & \\
\hline \multirow[t]{2}{*}{7} & Step0 & 12 & 8604 & 42101 & 0.057 & 2 \\
\hline & Final Step & 10.44 & 8604 & 48998.97 & 0.0129 & \\
\hline \multirow[t]{2}{*}{8} & Step0 & 13 & 5705 & 35818 & 0.078 & 6 \\
\hline & Final Step & 11.05 & 5705 & 44397.74 & 0.069 & \\
\hline \multirow[t]{2}{*}{10} & Step0 & 22 & 50726 & 34340 & 0.108 & 7 \\
\hline & Final Step & 10.57 & 50726 & 59033 & 0.031 & \\
\hline \multirow[t]{2}{*}{11} & Step0 & 19 & 34172 & 58424 & 0.022 & 3 \\
\hline & Final Step & 14.31 & 34172 & 77762.34 & 0.023 & \\
\hline \multirow[t]{2}{*}{12} & Step0 & 21 & 13320 & 63294 & 0.034 & 3 \\
\hline & Final Step & 15.82 & 13320 & 76585.74 & 0.041 & \\
\hline \multirow[t]{2}{*}{13} & Step0 & 50 & 25721 & 103245 & -0.007 & 5 \\
\hline & Final Step & 32.81 & 25721 & 156615.78 & -0.009 & \\
\hline \multirow[t]{2}{*}{14} & Step0 & 20 & 16652 & 71818 & 0.032 & 3 \\
\hline & Final Step & 17.02 & 16652 & 87475.27 & 0.076 & \\
\hline \multirow[t]{2}{*}{15} & Step0 & 25 & 35332 & 92136 & 0.016 & 2 \\
\hline & Final Step & 20.25 & 35332 & 110737.24 & 0.020 & \\
\hline \multirow[t]{2}{*}{16} & Step0 & 46 & 42185 & 101144 & 0.014 & 5 \\
\hline & Final Step & 28.5 & 42185 & 162893.42 & 0.011 & \\
\hline \multirow[t]{2}{*}{17} & Step0 & 44 & 81238 & 170336 & 0.007 & 2 \\
\hline & Final Step & 35.64 & 81238 & 196317.40 & 0.009 & \\
\hline \multirow[t]{2}{*}{18} & Step0 & 39 & 56396 & 146758 & 0.011 & 2 \\
\hline & Final Step & 32.27 & 56396 & 177577.18 & 0.010 & \\
\hline \multirow[t]{2}{*}{22} & Step0 & 10 & 19521 & 47231 & 0.241 & 1 \\
\hline & Final Step & 9.78 & 19521 & 51954.10 & 0.246 & \\
\hline \multirow[t]{2}{*}{24} & Step0 & 21 & 6293 & 3048 & 0.111 & 6 \\
\hline & Final Step & 13.47 & 6293 & 54011.35 & 0.058 & \\
\hline
\end{tabular}

\section{Conclusion}

In this paper, we developed a target setting algorithm to produce a path of targets for each inefficient unit. The RTS category of all targets in the sequence is the same as the inefficient units. Also, the efficiency score of the targets in the path are ascending and the 
final target is efficient. In other words, we suggest a sequence of targets, all similar to the unit under evaluation in size and we gradually improve the efficiency scores until we reach the efficient frontier. Such improvement is meaningful and feasible in practice. At each step, upper and lower bounds for the variation of each input and output of the inefficient unit are parameters that can be characterized according to the DM's opinion regarding the managerial restrictions. Based on this step size, we can select the similar target close to the efficient frontier as much as possible.

The number of steps to reach the final boundary point depends on the defined lower and upper bounds. Also if the unit is closer to the intersection of two regions with different returns to scale category, the number of steps are increased. This problem can not be prohibited because it is caused due to the location of units in the production possibility set. Our empirical example shows that the performance of the proposed target setting algorithm is efficient. Finally, one idea for the furture research is how this algorithm can be extended to other types of production possibility sets. Besides, the authors would like to consider the case of multiple optimal solutions. Also, finding a way to determine upper and lower bounds for each inefficient unit mathematically and according to the observed data can be one of important and intersting issues to be considered in the future.

\section{References}

Banker, R. D., Charnes, A., Cooper, W. W. (1984). Some models for estimating technical and scale inefficiencies in data envelopment analysis. Management science, 30(9), 1078-1092.

Brockett, P. L., Rousseau, J. J., Wang, Y., Zhow, L. (1997). Implementation of DEA models using GAMS. Research Report 765, University of Texas, Austin.

Charnes, A., Cooper, W. W., Rhodes, E. (1978). Measuring the efficiency of decision making units. European journal of operational research, 2(6), 429-444.

Coelli, T. (1998). A multi-stage methodology for the solution of orientated DEA models. Operations Research Letters, 23(3), 143-149.

Cooper, W. W., Park, K. S., Pastor, J. T. (1999). RAM: a range adjusted measure of inefficiency for use with additive models, and relations to other models and measures in DEA. Journal of Productivity analysis, 11(1), 5-42.

Cooper, W. W., Seiford, L. M., Tone, K. (2006). Data envelopment analysis. a comprehensive text with models, applications, references and dea-solver software. Springer, New York. 
Estrada, S. A., Song, H. S., Kim, Y. A., Namn, S. H., Kang, S. C. (2009). A method of stepwise benchmarking for inefficient DMUs based on the proximity-based target selection. Expert Systems with Applications, 36(9), 11595-11604.

Färe, R., Grosskopf, S. (1985). A nonparametric cost approach to scale efficiency. The Scandinavian Journal of Economics, 594-604.

Frei, F. X., Harker, P. T. (1999). Projections onto efficient frontiers: theoretical and computational extensions to DEA. Journal of Productivity analysis, 11(3), 275-300.

Lins, M. E., Angulo-Meza, L., Da Silva, A. M. (2004). A multi-objective approach to determine alternative targets in data envelopment analysis. Journal of the Operational Research Society, 55(10), 1090-1101.

Lozano S, Calzada-Infante L. (2017). Computing gradient-based stepwise benchmarking paths. Omega.

Lozano, S., Villa, G. (2005). Determining a sequence of targets in DEA. Journal of the operational research society, 56(12), 1439-1447.

Nasrabadi, N., Dehnokhalaji, A., Soleimani-Damaneh, M. (2014). Characterizing a subset of the PPS maintaining the reference hyperplane of the radial projection point. Journal of the Operational Research Society, 65(12), 1876-1885.

Park, J., Bae, H., Lim, S. (2012). A DEA-based method of stepwise benchmark target selection with preference, direction and similarity criteria. International Journal of Innovative Computing, Information and Control, 8(8), 5821-5834.

Pastor, J. T., Ruiz, J. L., Sirvent, I. (1999). An enhanced DEA Russell graph efficiency measure. European Journal of Operational Research, 115(3), 596-607.

Russell, R. R. (1985). Measures of technical efficiency. Journal of Economic theory, 35(1), 109-126.

Seiford, L. M., Thrall, R. M. (1990). Recent developments in DEA: the mathematical programming approach to frontier analysis. Journal of econometrics, 46(1-2), 7-38.

Tone, K. (2001). A slacks-based measure of efficiency in data envelopment analysis. European journal of operational research, 130(3), 498-509. 